\title{
Hydraulic Modeling of an Automatic Upstream Water Level Control Gate for Submerged Flow Conditions
}

\author{
Gilles BELAUD, Xavier LITRICO ${ }^{\dagger}$, Bertus DE GRAAFF ${ }^{\ddagger}$, Jean-Pierre BAUME ${ }^{\S}$
}

May 31, 2007

\begin{abstract}
The article proposes a mathematical model of an automatic upstream water level control gate designed to operate under free and submerged flow conditions, called the Vlugter gate. This automatic gate controls the upstream level close to a design level, using a counterweight to compensate for the hydraulic pressure on the gate. The proposed gate model is an extension of an existing model for free flow conditions (Begemann gate) to submerged conditions. Submergence effects are introduced both in the discharge law and the equilibrium law. The mathematical model is validated on experimental data from two small-scale gates and on data from literature, in order to show the ability of the model to simulate the behavior of gates of various dimensions.
\end{abstract}

Keywords: upstream control, gate, hydraulic model, analytical model, submerged flow, laboratory tests.

\section{Introduction}

Flap gates are used in many open-channel networks to control upstream water levels. The article deals with a specific type of gate, initially described by Vlugter (1940), and more recently mentioned by Brouwer (1987), Brants (1996), de Graaff (1998), Burt et al. (2001) and Litrico et al. (2005). Such gates are installed in several irrigation projects, located in developing countries such as Nigeria and Indonesia. They are used as an affordable mechanical means to control water distribution, generally in conjunction with orifices or baffle modules to deliver a desired discharge in the distributary channels. Begemann and Vlugter gates were installed in two Nigerian irrigation schemes built in the 1970's (Brouwer, 1987): the Kano River Irrigation Project (KRIP) and the Hadejia Valley Irrigation Project (HVIP). Four cross regulators of the HVIP North Main Canal are equipped with a series of Vlugter and Begemann gates, and the water distribution in the secondary channels of KRIP is performed with similar gates in conjunction with baffle modules.

The considered automatic gate, the Vlugter gate, consists of a weir equipped with a steel plate rotating around a horizontal axis located above the upstream water level (see Fig. 1). When

\footnotetext{
${ }^{*}$ Associate Professor, ENSAM, 2 place Viala, 34060 Montpellier, France, e-mail: belaud@ensam.inra.fr

${ }^{\dagger}$ Researcher Hydraulic Engineer, Cemagref, UMR G-EAU, B.P. 5095, 34196 Montpellier Cedex 5, France, e-mail: xavier.litrico@cemagref.fr

${ }^{\ddagger}$ Researcher hydrology, HKV consultants, Botter 11-29, 8232 JN, Lelystad, The Netherlands, e-mail: b.de.graaff@hkv.nl

${ }^{\S}$ Researcher Hydraulic Engineer, Cemagref, e-mail: jean-pierre.baume@cemagref.fr
} 
the gate is open, water flows under the plate but also on its sides. A counterweight on the top of the plate compensates for the hydraulic pressure exerted by the water. When the upstream water level increases, the pressure also increases and the corresponding moment tends to open the gate. When the water level decreases, the pressure diminishes and the moment exerted by the counterweight tends to close the gate. The equilibrium is obtained when the closing moment exerted by the weight of the gate compensates for the opening moment exerted by the hydraulic pressure. When properly designed, such a gate can maintain upstream levels within a small range around the design upstream level (see Vlugter (1940); Burt et al. (2001)). Litrico et al. (2005) proposed a model to compute the water level and the gate opening for this type of gates functioning in free flow conditions (Begemann or flap gates), based on discharge and equilibrium relationships.

However, submerged conditions may be encountered in flat canals, where cross structures must be designed with little head loss. This paper deals with a modified version of the Begemann gate called the Vlugter gate, which is equipped with a cylindrical float on the downstream side of the gate to limit the downstream influence on the gate. The hydraulic modelling of such gates is rather challenging, mainly due to the flow configuration which is changing with the gate opening: for high opening angles, the gate behaves as a weir, while for small opening angles, its behavior is close to an orifice.

To derive a mathematical model, these gates were observed in the field during two missions in Nigeria and two small-scale laboratory gates have been designed and used for experimental data acquisition: a reduced scale experimental gate was built in the laboratory canal of ENSAM (Ecole Nationale Supérieure Agronomique de Montpellier, France), and another one was built in the laboratory canal of Delft University of Technology, which we will refer to as the Delft gate.

The first model developed by de Graaff (1998) based on the momentum conservation principle was not able to accurately reproduce the gate behaviour for high flows and for high submergence ratios. This paper proposes an extension of the model of Litrico et al. (2005) to the submerged conditions for Vlugter gates, introducing submergence effects both in the discharge law and the equilibrium law.

The article is structured as follows: we first describe the behavior of the gate, then we present the experimental setup. In the next section, we derive the model of the Vlugter gate, with

first the discharge law, then the equilibrium law and finally the combination of both in order to predict the upstream water level and gate opening at equilibrium.

\section{System Description and Behavior}

\section{Gate Description}

The gate designed by Vlugter consists of a flap gate equipped with a float on the downstream side. The float is a portion of a cylinder centered around the pivot line (point O, see Fig. 1) so that the moment exerted by the downstream water body is null, since the lever arm of the downstream pressure force is null.

The design water level, denoted $h_{d}$, is the maximum upstream level in closed position. It may be easily adjusted thanks to the counterweight. The location of the center of gravity, G, is usually referred to by the angle $\Phi$, which is the angle between the horizontal and the plane defined the pivot line and G (see Fig. 1). The angle $\Phi$ has a large influence on the deviation from the design water level. A larger $\Phi$ results in a more sensitive gate and the sensitivity may 
be so large that the gate may become unstable for large values of $\Phi$ (de Graaff, 1998). Some gates are equipped with dampers to reduce possible oscillations.

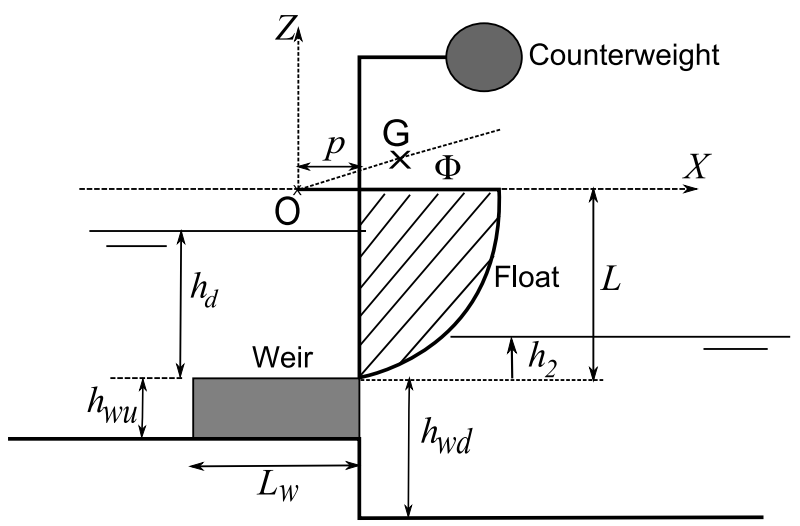

Figure 1: Vlugter gate, closed position

This study is based on experiments conducted in Delft (de Graaff, 1998) and ENSAM hydraulic laboratories with two different gates. Vlugter's original data were used for the model validation (Vlugter, 1940). The characteristics of the gates are given in table 1. We denote in the following: $L$ the gate height (see Fig. 1), $B_{w}$ the weir width, $B_{g}$ the gate width, $L_{w}$ the weir length, $p$ the lever arm (distance between the pivot and the plate) and $M$ the total mass of the gate. The weir upstream and downstream heights are denoted $h_{w u}$ and $h_{w d}$ respectively. Note that $B_{g}$ is slightly greater than $B_{w}$ : in closed position, the contact width between the gate and the weir is $1.5 \mathrm{~cm}$ on each edge for ENSAM gate and $2 \mathrm{~cm}$ for Delft gate.

Table 1: Gate dimensions, Delft and ENSAM small scale gates. The horizontal and vertical coordinates are denoted $X$ and $Z$ (see Fig. 1)

\begin{tabular}{|l|c|c|c}
\hline \hline Specifications & Delft gate & ENSAM gate & $\begin{array}{c}\text { Vlugter gate } \\
\text { (original data) }\end{array}$ \\
\hline Weir length $B_{w}$ & $0.40 \mathrm{~m}$ & $0.38 \mathrm{~m}$ & $6 \mathrm{~m}$ \\
Gate width $B_{g}$ & $0.44 \mathrm{~m}$ & $0.40 \mathrm{~m}$ & $6 \mathrm{~m}$ \\
Gate height $L$ & $0.47 \mathrm{~m}$ & $0.45 \mathrm{~m}$ & $1.5 \mathrm{~m}$ \\
Horizontal lever arm $p$ & $0.11 \mathrm{~m}$ & $0.045 \mathrm{~m}$ & $0.6 \mathrm{~m}$ \\
Total mass $M$ & $41.5 \mathrm{~kg}$ & $24.1 \mathrm{~kg}$ & $6350 \mathrm{~kg}$ \\
Angle $\Phi$ & $\{35,45,55\}$ deg & $\{38,45,58\} \mathrm{deg}$ & $45 \mathrm{deg}$ \\
Center of gravity position $\mathrm{G}$ & $X_{G}=0.15 \mathrm{~m}, Z_{G}$ variable & $X_{G}$ variable, $Z_{G}=0.14 \mathrm{~m}$ & $X_{G}=0.7 \mathrm{~m}$ \\
\hline \hline
\end{tabular}

\section{Experimental Setup}

\section{ENSAM Gate}

The gate is placed in the middle of a $25 \mathrm{~m}$ long and $0.70 \mathrm{~m}$ wide flume. The discharge in the flume is controlled by baffle weir modules. The downstream water level can be adjusted with an undershot gate. 


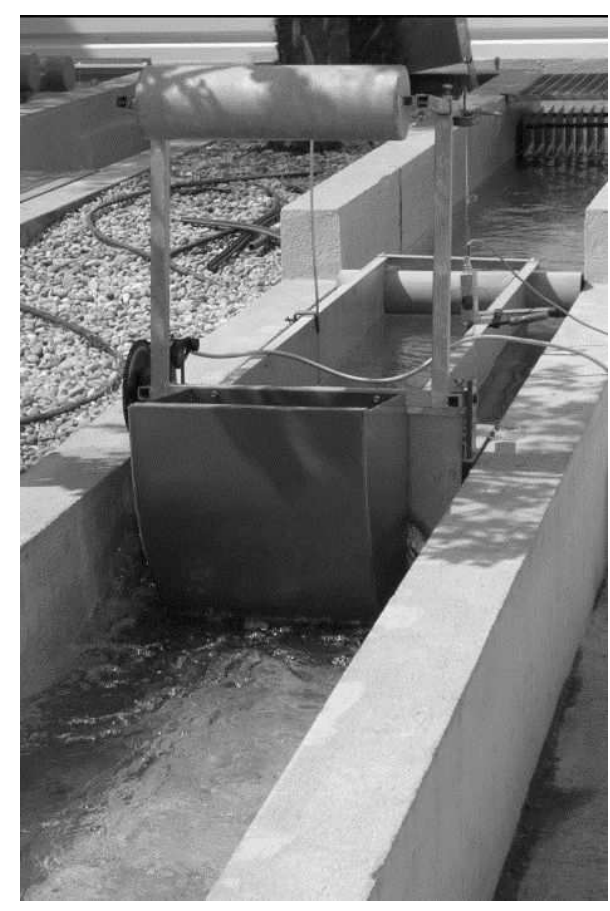

Figure 2: ENSAM gate

Three counterweight positions were studied ( $\Phi=38,45$ and 58 degrees). For each counterweight position, three discharges were imposed (low $Q=10 \mathrm{l} / \mathrm{s}$, intermediate $Q=30 \mathrm{l} / \mathrm{s}$, and maximum discharge $Q=60 \mathrm{l} / \mathrm{s}$ ). For each discharge condition, the downstream water level $h_{2}$ was increased progressively from 0 or even below (free flow) to a maximum value of around 30 $\mathrm{cm}$ (canal overtopping, depending on the discharge). In the latter case, the gate was always fully open. For each value of $\left(\Phi, Q, h_{2}\right)$, the upstream water level $h_{0}$ and the gate opening angle $\delta$ at equilibrium were measured by sensors at a time step of 1 second.

Some unstable behavior appeared, especially at low discharge. Such behaviors are not addressed in this paper. Finally, 2 to 10 measurements were available for each combination of counterweight position and discharge for a total of 44 data sets.

The weir behavior (gate removed) was also studied with 7 discharge values from 10 to $60 \mathrm{l} / \mathrm{s}$.

\section{Delft Gate}

The gate is placed at the downstream end of a $39 \mathrm{~m}$ long, $0.80 \mathrm{~m}$ wide and $0.80 \mathrm{~m}$ deep flume. The discharge is controlled by tuning the opening of a pipe connecting the flume and a large water tank located above the flume. The head in the tank is maintained constant during the experiments. The discharges are determined from the static pressure head over an standardized orifice meter. Three counterweight positions were studied ( $\Phi=35,45$ and 55 degrees). For each counterweight position the discharge was increased in four stages, from a low discharge of $45 \mathrm{l} / \mathrm{s}$ up to a maximum discharge of $166 \mathrm{l} / \mathrm{s}$ depending on the counterweight position and the water level downstream of the gate. The downstream water level was increased progressively from below the weir level up to a maximum of $29 \mathrm{~cm}$ above the weir level, again depending on the counterweight position and the water level downstream of the gate.

Unstable behavior was encountered for $\Phi$ angles of 45 and 55 degrees for low flows. These instabilities are not addressed in this paper. Finally, a total of 36 data sets is available on this 


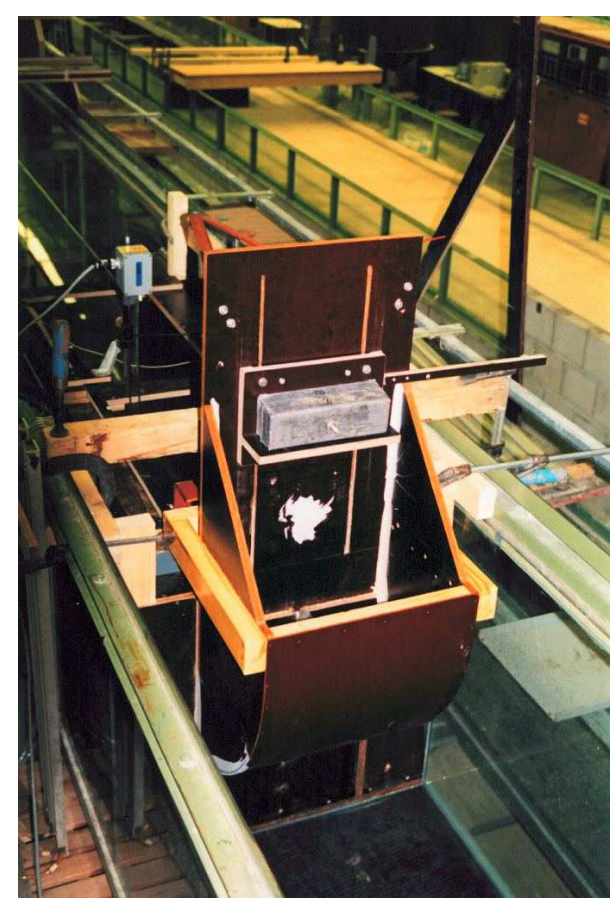

Figure 3: Delft gate

gate.

The weir behavior was also studied with 7 discharge values from 45 to $170 \mathrm{l} / \mathrm{s}$.

\section{Mathematical Model of the Head-Opening-Discharge Relation- ship}

\section{Basic principle}

The model derived here calculates the discharge for given values of the upstream and downstream levels $\left(h_{0}\right.$ and $\left.h_{2}\right)$ and opening angle $\delta$. The angle $\delta$ may differ from the equilibrium angle $\delta^{*}$, by moving the gate manually for instance. Following the idea introduced in Litrico et al. (2005) for the Begemann gate, we consider that the gate behaves as an undershot gate at small openings, and as a broad-crested weir at large openings. Between these regimes, the variation of the discharge $Q$ with the opening angle is calculated by a cubic polynomial interpolation. The value of $Q(\delta)$ and its first order derivative is calculated in two characteristic points.

\section{Calculation for Small Angles $\delta$}

When the gate is closed $(\delta=0)$, the discharge equals 0 or a residual discharge $Q_{r}$ if any. To analyze the gate behavior, we also search for the derivative of $Q$ with respect to $\delta$. We first consider the free flow behavior, then we analyze the effect of submergence. 


\section{Calculation in free flow}

Such as for Begemann gate (see Litrico et al. (2005)), the flow can be divided into two parts, one part flowing under the gate $\left(Q_{u}\right.$, area $\left.A_{u}\right)$ behaving as a flow through an orifice, and one part flowing on the sides $\left(Q_{s}\right)$, behaving as a flow over a weir. In the following, the subscript $f$ denotes for the free flow condition.

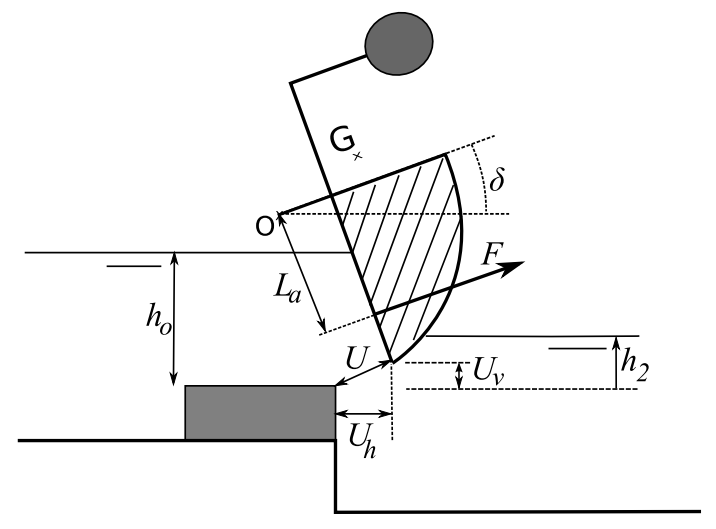

Figure 4: Vlugter gate, open position

Discharge under the gate, $Q_{u f}-$ When $\delta$ increases, the discharge can be evaluated by applying Bernoulli's formula between the upstream reach and the contracted section below the gate. This yields the classical discharge formula (Bos (1989)):

$$
Q_{u f}=C_{c} A_{u} \sqrt{2 g\left(H_{0}-h_{1}\right)}
$$

where $A_{u}$ is the opening area under the gate, $C_{c}$ is the contraction coefficient, $g$ the gravitational acceleration, $H_{0}$ the total upstream head and $h_{1}$ is the hydrostatic head at the contracted section. In free flow, the experiments showed that the value of $h_{1}$ is small compared to $H_{0}$. Eq. 1 is usually simplified as follows:

$$
Q_{u f}=C_{u} A_{u} \sqrt{2 g h_{0}}
$$

where $C_{u}$ is a discharge coefficient, close to the contraction coefficient $C_{c}$ and taking account for the approximation $H_{0}-h_{1} \simeq h_{0}$.

$A_{u}$ is calculated by multiplying the gate width, $B_{g}$, by the distance $U$ between the bottom lip of the gate and the weir downstream edge. We obtain:

$$
\sin (\delta / 2)=\frac{U / 2}{L^{2}+p^{2}}
$$

and finally

$$
A_{u}=2 B_{g} \sqrt{L^{2}+p^{2}} \sin (\delta / 2)
$$

From Eq. 2, we calculate the derivative of $Q_{u f}$ in 0 :

$$
\frac{\partial Q_{u f}}{\partial \delta}(0)=C_{u} \sqrt{2 g h_{0}} \frac{\partial A_{u}}{\partial \delta}(0)
$$

where

$$
\frac{\partial A_{u}}{\partial \delta}(0)=B_{g} \sqrt{L^{2}+p^{2}}
$$




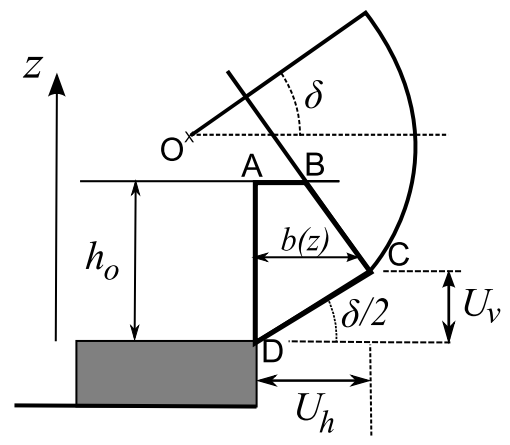

Figure 5: Flow geometry, side discharge

Discharge on the sides, $Q_{s f}$ - The corresponding discharge $Q_{s f}$ is the one of a weir, the shape of which is illustrated on Fig. 5 (polygon ABCD). This discharge is obtained by integrating the velocity, $\sqrt{2 g\left(h_{0}-z\right)}$, on the flow section (ABCD), where $z$ is the height above the sill. The weir width, denoted $b(z)$, is defined by:

$$
\begin{aligned}
& z<U_{v} \quad \Rightarrow \quad b(z)=\tan (\delta / 2) z \\
& z \geq U_{v} \Rightarrow b(z)=U_{h}-\tan (\delta)\left(z-U_{v}\right)
\end{aligned}
$$

where $U_{v}=L(1-\cos \delta)+p \sin \delta$ is the vertical opening, and $U_{h}=p(\cos \delta-1)+L \sin \delta$ is the horizontal opening (see Fig. 5). The flow is contracted, and it can be assumed that the contraction coefficient is the same as for the flow under the gate, $C_{c}$, at least for small openings. We have

$$
\begin{aligned}
Q_{s f} & =2 C_{c} \int_{0}^{h_{0}} b(z) \sqrt{2 g\left(h_{0}-z\right)} d z \\
& =2 \sqrt{2 g} C_{c}\left[\tan \left(\frac{\delta}{2}\right) \int_{0}^{U_{v}} z \sqrt{h_{0}-z} d z+\int_{U_{v}}^{h_{0}}\left(U_{h}-\tan (\delta)\left(z-U_{v}\right)\right) \sqrt{h_{0}-z} d z\right]
\end{aligned}
$$

which integrates in

$$
\begin{aligned}
Q_{s f}= & 2 C_{c} \sqrt{2 g}\left[\left(\frac{4}{15} h_{0}^{5 / 2}-\frac{2}{5}\left(h_{0}-U_{v}\right)^{3 / 2}\left(\frac{2}{3} h_{0}+U_{v}\right)\right) \tan \left(\frac{\delta}{2}\right)\right. \\
& \left.+\frac{2}{3}\left(U_{h}+U_{v} \tan (\delta)\right)\left(h_{0}-U_{v}\right)^{3 / 2}-\frac{2}{5} \tan (\delta)\left(h_{0}-U_{v}\right)^{3 / 2}\left(\frac{2}{3} h_{0}+U_{v}\right)\right]
\end{aligned}
$$

The derivative in $\delta=0$ is obtained by expanding the above expression in Taylor series, which yields

$$
\frac{\partial Q_{s f}}{\partial \delta}(0)=2 \sqrt{2 g} C_{c} \frac{2}{3} h_{0}^{3 / 2}\left(L-\frac{2}{5} h_{0}\right)
$$

\section{Calculation in submerged conditions}

In this case, $h_{2}>0$. We first consider the discharge under the gate, $Q_{u}$. Applying Bernoulli's principle, equation 1 is still valid, but the head $h_{1}$ is close to the downstream water level $h_{2}$. Equation 1 writes as follows:

$$
Q_{u}=C_{u} A_{u} \sqrt{2 g h_{0}(1-s)}
$$

where $s=h_{2} / h_{0}$ is the submergence ratio. In other terms, the discharge is multiplied by a reduction factor due to submergence:

$$
S(s)=\sqrt{1-s}
$$


and so it is for the derivative $\partial Q_{u} / \partial \delta(0)$.

The same can be done for the side discharge, $Q_{s}$. This discharge is obtained by multiplying the side discharge in free flow, $Q_{s f}$, by a reduction factor $S$, such as usually done for submerged weirs (see (Sinniger and Hager, 1989; USDA, 1973; Hager and Schwalt, 1994; Bos, 1989) among others). In practice, it is difficult to estimate separately the flow under the gate from the flow on the sides and therefore to determine the modular limit and the reduction factor for the side flow, which is not a usual weir. Different functions $S(s)$ were tested for the side flow, but little difference was observed between the different models. This is due to the fact that the discharge under the gate accounts for the major part of the total flow.

Considering that the discharge relations for the flow under the gate and on the sides cannot be calibrated separately, we choose the same submergence function $S(s)=\sqrt{1-s}$ and the same contraction coefficient, $C_{c} \simeq C_{u}$, for both $Q_{u}$ and $Q_{s}$. The combination of Eq. 5, 6, 14 and 12 gives the derivative of $Q$ in $\delta=0$ :

$$
\frac{\partial Q}{\partial \delta}(0)=C_{u} \sqrt{2 g h_{0}(1-s)}\left[B_{g} \sqrt{L^{2}+p^{2}}+\frac{2}{3} h_{0}\left(2 L-\frac{4}{5} h_{0}\right)\right]
$$

where $C_{u}$, discharge coefficient of the gate, should be around 0.6 to 0.9 . The discharge coefficients were obtained by calibration on the discharge curve data, leading to $C_{u}=0.85$ for both gates.

\section{Weir Behavior}

\section{Weir formula}

Here we analyze the flow when the gate is largely open. The gate has little influence on the flow, therefore we first consider the discharge through a weir, denoted $Q_{w}$.

For free flow, the classical formulation is used (Hager and Schwalt, 1994; Bos, 1989):

$$
Q_{w f}=C_{w} B_{w} \sqrt{2 g} H_{0}^{3 / 2}
$$

in which $Q_{w f}$ denotes the discharge through the weir in free flow, $H_{0}$ the total upstream head above the weir, $C_{w}$ the weir discharge coefficient. This coefficient, close to 0.385 , can be expressed as a function of the weir length $L_{w}$, the upstream head $H_{0}$, the weir upstream height $\left(h_{w u}\right)$ or downstream height $\left(h_{w d}\right)$. For a broad-crested weir, a simple formulation is adopted (Sinniger and Hager, 1989; Hager and Schwalt, 1994):

$$
C_{w}=C_{w_{0}}\left(1-\frac{2}{9\left(1+\left(H_{0} / L_{w}\right)^{4}\right)}\right)
$$

in which $L_{w}$ is the weir length and $C_{w_{0}}$ is a constant discharge coefficient.

As introduced before, submerged weirs can be modeled by using the free weir discharge equation and a reduction factor $S=Q_{w} / Q_{w f}$ due to submergence. A classical formulation is used (see (Sinniger and Hager, 1989; Hager and Schwalt, 1994))

$$
\begin{aligned}
s_{1} & =\left(s-y_{L}\right) /\left(1-y_{L}\right) \\
S_{w} & =\left(1-s_{1}^{m_{1}}\right)^{m_{2}}
\end{aligned}
$$

where $y_{L}$ is the modular limit of the weir (maximum value of $s=h_{2} / h_{0}$ for the free flow behavior), $m_{1}$ and $m_{2}$ coefficients defined earlier. Such as suggested by Hager and Schwalt (1994) and Sinniger and Hager (1989), the values $C_{w_{0}}=0.435, y_{L}=0.75, m_{1}=2$ and $m_{2}=0.5$ gave good results on the laboratory gates. Note that the weir behavior can be easily calibrated by removing or opening manually the gate. 


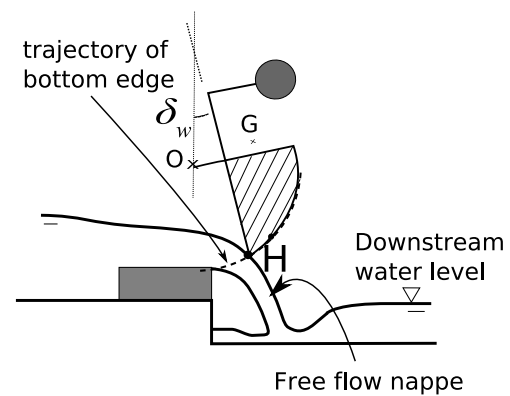

case 1: free weir, $h_{2}<0$

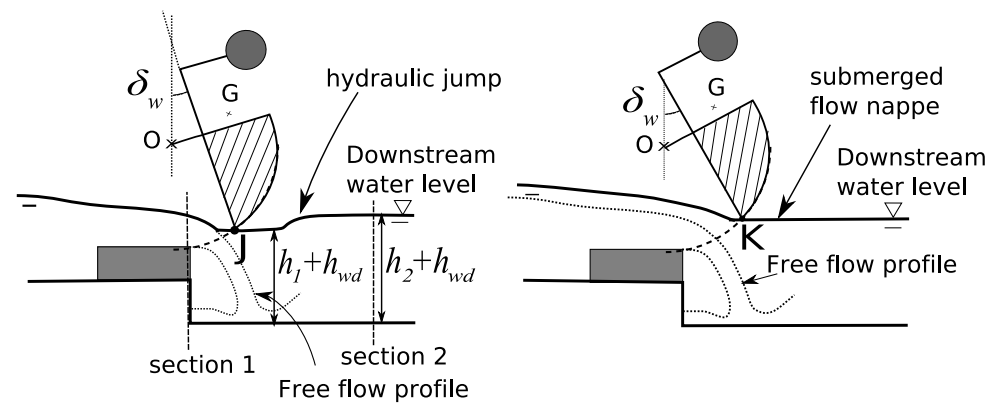

case 2: free weir, $0<h_{2} / h_{0}<y_{L}$

case 3: submerged weir, $h_{2} / h_{0}>y_{L}$

Figure 6: Influence of downstream level on $\delta_{w}$

\section{Calculation of $\delta_{w}$}

The discharge through the gate equals $Q_{w}$ as long as $\delta$ is greater than or equal to $\delta_{w}$, angle for which the bottom lip of the gate touches the upper nappe of the flow. This angle is therefore obtained by computing the intersection of the circle described by the bottom edge of the gate and the upper nappe profile. The computation of the upper nappe is then subject to three different cases (see Fig. 6):

- When the downstream level is low $\left(h_{2}<0\right)$, the problem consists in determining the upper nappe profile generated by the free overfall (case 1 ).

- For higher downstream levels, but still in free flow, the circle described by the bottom edge of the gate may touch the free surface downstream from the jet issuing from the free overfall. In this case, the contact point is between the jet and the hydraulic jump transforming the kinetic energy of the jet into potential energy (case 2);

- When the weir is submerged (say, $s=h_{2} / h_{0}$ is above the modular limit $y_{L}$ ), the upper nappe profile is modified (case 3 ).

Case 1: free flow, $h_{2} \leq 0$ : Such as for Begemann gate, the method proposed by Hager (1983) and Davis et al. (1999) was used to derive the equation of the upper nappe:

$$
z_{\text {nappe }}=h_{e}-x \tan \alpha-x^{2} \frac{g}{2 V_{u}^{2} \cos ^{2} \alpha}
$$

with $h_{e}$ brink depth (taken equal to 0.7 times the critical depth), $V_{u}$ flow velocity through the weir, $\alpha$ angle of the flow with the horizontal at the brink, $x$ the horizontal distance from the brink and $z$ the vertical abscissa taken from the sill. This angle is very small and is taken equal to $0^{\circ}$, implying $V_{u}=Q /\left(B_{w} h_{e}\right)$.

This profile is intersected with the trajectory of the bottom edge of the gate, which is a circle of radius $\sqrt{L^{2}+p^{2}}$ centered on the pivot line. The intersection calculation is made by a classical zero-function search method and yields the value of $\delta_{w}$. The coordinates of the intersection point $\mathrm{H}$ (see Fig. 6) are denoted $\left(x_{H}, z_{H}\right)$.

Case 2: free flow, $y_{L} h_{0} \geq h_{2}>0$ : The downstream influence is now considered. Since the flow through the weir is supercritical, a hydraulic jump occurs below the gate. The water level 
below the gate can be roughly estimated by applying the momentum conservation principle between the brink (downstream end of the weir, section 1) and section 2 (downstream pool, see Fig. 6).

The momentum of the jet, at the brink section, equals $M_{1}=\rho Q V_{u}=\rho Q^{2} /\left(B_{w} h_{e}\right)$. In section 2 , it equals $M_{2}=\rho Q^{2} /\left(B_{c}\left(h_{2}+h_{w d}\right)\right)$, where $B_{c}$ is the canal width in the downstream pool. The forces exerted on the fluid are the pressure forces in section 1 and $2, F_{p 1}$ and $F_{p 2}$. In section 1 , the water depth is $h_{1}+h_{w d}$ in the downstream pool and $h_{e}$ in the jet issuing from from the weir. The pressure force is given by

$$
F_{p 1}=\frac{1}{2} \rho g B_{c}\left(h_{1}+h_{w d}\right)^{2}+\frac{1}{2} \rho g B_{w} h_{e}^{2}
$$

Similarly, $F_{p 2}=\frac{1}{2} \rho g B_{c}\left(h_{2}+h_{w d}\right)^{2}$. The momentum principle writes as follows:

$$
M_{1}+F_{p 1}=M_{2}+F_{p 2}
$$

and

$$
\frac{1}{2} \rho g B_{w} h_{e}^{2}+\rho Q^{2} /\left(B_{w} h_{e}\right)+\frac{1}{2} \rho g B_{c}\left(h_{1}+h_{w d}\right)^{2}=\rho Q^{2} /\left(B_{c}\left(h_{2}+h_{w d}\right)\right)+\frac{1}{2} \rho g B_{c}\left(h_{2}+h_{w d}\right)^{2}
$$

which gives

$$
h_{1}=\sqrt{\left(h_{2}+h_{w d}\right)^{2}+\frac{B_{w}}{B_{c}} h_{e}^{2}-\frac{Q^{2}\left[\frac{1}{B_{w} h_{e}}-\frac{1}{B_{c}\left(h_{2}+h_{w d}\right)}\right]}{g B_{c}}}-h_{w d}
$$

At low discharge, both upstream and downstream momenta are low, and the same for $h_{e}$, calculated from the critical depth. From Eq. (24), it can be observed that $h_{1} \simeq h_{2}$. When the discharge increases, $h_{2}$ being fixed, the momentum difference increases and the water level below the gate decreases. This was indeed observed during experiments.

Two situations may be observed:

- If $h_{1}<z_{H}$, the angle $\delta_{w}$ is not affected by the downstream level and this case is equivalent to case 1 ;

- Otherwise the gate touches the water downstream from the nappe created by the overfall. We assume that the intersection appears upstream from the hydraulic jump, where the water depth is $h_{1}$. In this case, the intersection point J must verify:

$$
\begin{aligned}
& z_{J}=h_{1} \\
& z_{J}=L\left(1-\cos \left(\delta_{w}\right)\right)+p \sin \left(\delta_{w}\right)
\end{aligned}
$$

Introducing $\theta=\tan \left(\delta_{w} / 2\right)$ and eliminating $z_{J}$, we get

$$
\delta_{w}=2 \arctan \left(\frac{\sqrt{p^{2}+L^{2}-\left(L-h_{1}\right)^{2}}-p}{2 L-h_{1}}\right)
$$

which is valid as long as $0 \leq h_{1}<2 L$. Let us remark that any level greater than $L+p$, which corresponds to $\delta=\pi / 2$, is unrealistic. 
Case 3: submerged flow, $y_{L} h_{0}<h_{2}$ : No hydraulic jump occurs. The water elevation is $h_{2}$ in the downstream pool (except in the close vicinity of the brink). The intersection point K must verify:

$$
\begin{aligned}
& z_{K}=h_{2} \\
& z_{K}=L\left(1-\cos \left(\delta_{w}\right)\right)+p \sin \left(\delta_{w}\right)
\end{aligned}
$$

which is solved the same way as for case 2 :

$$
\delta_{w}=2 \arctan \left(\frac{\sqrt{p^{2}+L^{2}-\left(L-h_{2}\right)^{2}}-p}{2 L-h_{2}}\right)
$$

\section{Derivative of $Q$ with respect to $\delta$}

The only element missing for the cubic polynomial interpolation of the discharge formulas is the variation of the discharge with $\delta$. Observations showed that, such as for free flow, closing the gate when $\delta \approx \delta_{w}$ does not affect the total discharge through the gate: the decrease of the flow rate below the gate is mainly compensated by the increase of discharge on the sides. We assume that

$$
\frac{\partial Q}{\partial \delta}\left(\delta_{w}\right)=0
$$

\section{Full Discharge Equation}

Given $h_{0}$ and $h_{2}$, the total discharge law $\left.Q(\delta)\right|_{h_{0}, h_{2}}$ is obtained by a polynomial interpolation between 0 and $\delta_{w}$. Knowing $Q(0)=Q_{r}, Q\left(\delta_{w}\right)=Q_{w}, \frac{\partial Q}{\partial \delta}(0)$ and $\frac{\partial Q}{\partial \delta}\left(\delta_{w}\right)$, a third-degree polynomial is adjusted:

$$
Q\left(\delta, h_{0}, h_{2}\right)=Q_{r}+c_{1} \delta+c_{2} \delta^{2}+c_{3} \delta^{3}
$$

with $c_{1}, c_{2}, c_{3}$ and $c_{4}$ given in appendix.

\section{Validation of the discharge formula}

\section{Upper nappe calculation}

Measurements of the upper nappe over the weir are available on ENSAM gate only. Figure 7 shows the comparison between measurements and the model developed in this study. The model is very satisfying in free flow conditions and in fully submerged conditions. When the gate touches the nappe in the hydraulic jump created by the gate, differences up to 2 degrees can be found between model and measurements for the angle $\delta_{w}$. This is considered as a sufficient precision for the complete model of the gate. Actually, the sensitivity of the discharge to the opening angle is small in this domain, which is expressed in our method by Eq. (31).

\section{Discharge law}

In this section, the opening angles are calculated for each set of measured values $\left(Q, h_{0}, h_{2}\right)$. The results are compared to the measured values in Fig. 8. The openings are expressed as a ratio of the opening angle $\delta$ to the maximum opening angle $\delta_{\max }$ where $\delta_{\max }$ is the maximum angle of opening, corresponding to the center of gravity located exactly above the pivot:

$$
\delta_{\max }=\pi / 2-\Phi
$$




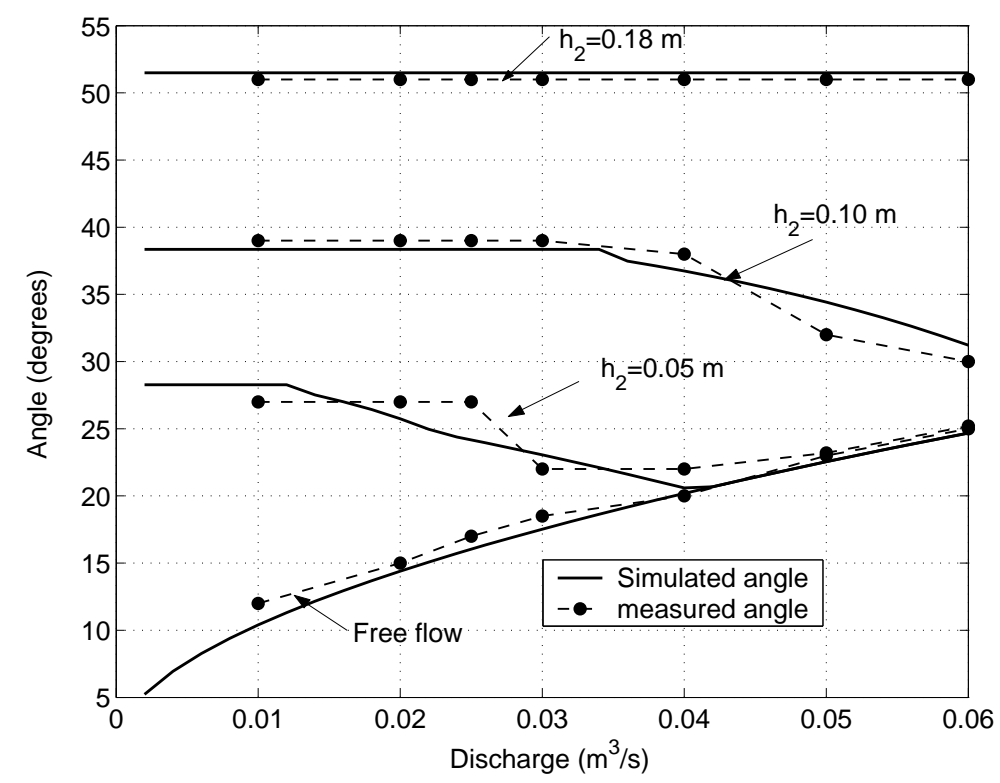

Figure 7: Comparison of model and measurements for the angle $\delta_{w}$ where the gate just touches the nappe, ENSAM gate.

The correspondence is fairly good, although the error increases at large angles. Indeed, the gate behaves as a weir at large angles and the flow is only slightly influenced by the gate opening, as expressed by the relation $\partial Q / \partial \delta=0$. Therefore, a small deviation in $Q, h_{0}$ or $h_{2}$ may dramatically change the predicted opening. Error margins were calculated for each measurement by calculating the angles with $\pm 5 \%$ of the measured discharge. It can be seen from figure 8 that the error margin increases with the opening and almost all the measured values are within the simulated interval. For example, the maximum error for ENSAM gate corresponds to $Q=30 \mathrm{l} / \mathrm{s}, \Phi=50^{\circ}$ and $h_{2} / h_{0}=0.69$. In this case, the gate was unstable $\left(\delta=\delta_{\max }=32^{\circ}\right)$. The predicted angle is $18^{\circ}$, but the calculated margin is [14-32 $]$.

\section{Mathematical Model of the Equilibrium State}

\section{Basic principle}

Due to the complexity of the pressure field and the shape of the free surface upstream of the gate, the force exerted on the plate is difficult to predict. Here, we analyse the trajectory of the force at equilibrium, denoted $F$, and we look for its expression with respect to the equilibrium angle $\delta^{*}$. F is obtained by interpolating between $\delta^{*}=0$, where the force is given by the hydrostatic assumption, and $\delta_{\max }=\pi / 2-\Phi$, where the gate is in unstable position, thus where $F=0$. The shape of the trajectory is assessed by the closing moment calculation:

$$
M_{c}\left(\delta^{*}\right)=M g\left(X_{G} \cos \left(\delta^{*}\right)-Y_{G} \sin \left(\delta^{*}\right)\right)
$$

where $X_{G}$ and $Z_{G}$ are the coordinates of the center of gravity in closed position. In all calculations, the lever arm is assumed to be as in hydrostatic conditions, i.e. at the $1 / 3$ of the wetted length of the gate. This wetted length is equal to $\left(h_{0}-U_{v}\left(\delta^{*}\right)\right) / \cos \left(\delta^{*}\right)$, where $U_{v}$ is 


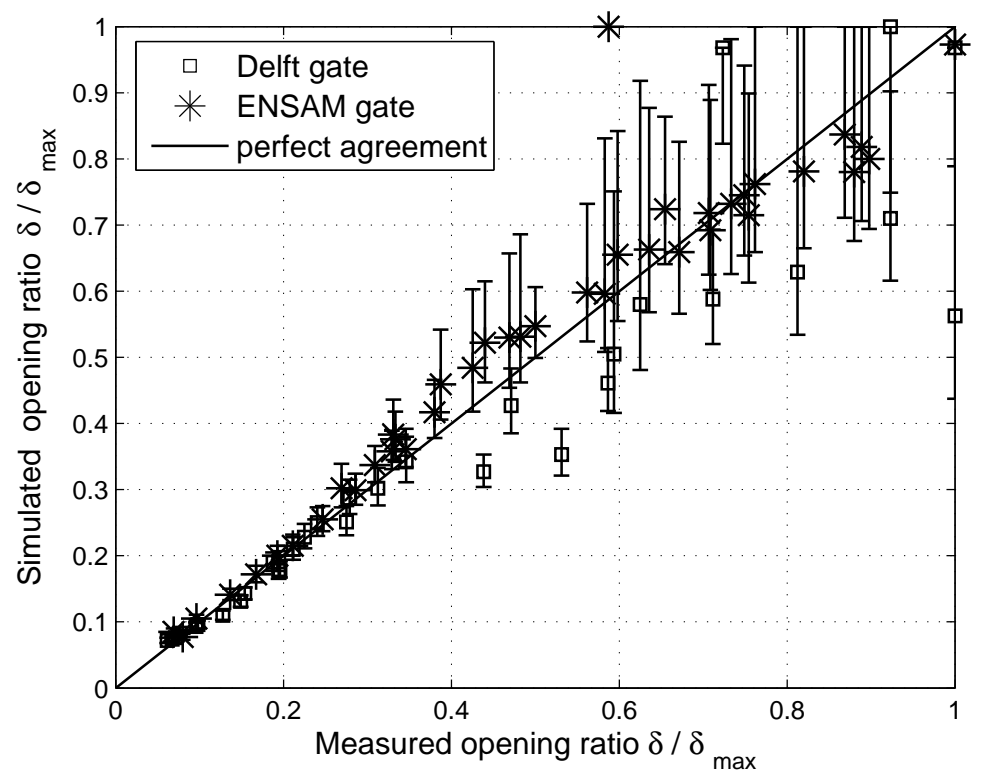

Figure 8: Predicted versus measured opening angles; for each measurement, the angle $\delta$ is divided by the maximum opening angle $\delta_{\max }$

the vertical opening of the gate (see Fig. 4), and the lever arm $L_{a}$ can be computed by:

$$
L_{a}\left(\delta^{*}, h_{0}\right)=L-\frac{h_{0}-U_{v}\left(\delta^{*}\right)}{3 \cos \left(\delta^{*}\right)}
$$

\section{Calculation near 0}

When the gate is closed $\left(\delta^{*}=0\right)$, the pressure field on the gate is hydrostatic. The force equals

$$
F(0)=\frac{1}{2} \rho g h_{d}^{2} B_{w}
$$

The derivative of the force in 0 is obtained by differentiating the following equation:

$$
F\left(\delta^{*}\right)=\frac{M_{o}\left(\delta^{*}, h_{0}\right)}{L_{a}\left(\delta^{*}, h_{0}\right)}
$$

Litrico et al. (2005) introduced and determined the non-dimensional coefficient $C_{F_{0}}=\left(\partial h_{0} / \partial \delta\right)(0) / h_{d}$, correlated with the angle $\Phi$, and obtained:

$$
\frac{d F}{d \delta}(0)=-\frac{M g}{L-\frac{h_{d}}{3}}\left(Y_{G}+\frac{p-C_{F_{0}} h_{d}}{3 L-h_{d}} X_{G}\right)
$$

\section{Calculation near $\delta_{\max }$}

For $\delta^{*}=\delta_{\max }$, the gate is in unstable equilibrium position, therefore the force should be equal to zero. The lever arm $L_{a}$ is equal to the gate length, To calculate the derivative of the force with respect to $\delta^{*}$, the opening moment is differentiated on the domain of equilibrium $M_{o}\left(h_{0}^{*}, \delta^{*}\right)=M_{c}\left(\delta^{*}\right)$ :

$$
\frac{d F\left(\delta^{*}\right)}{d \delta^{*}}=\frac{1}{L_{a}} \frac{d M_{o}}{d \delta^{*}}-\frac{M_{o}}{L_{a}^{2}} \frac{d L_{a}}{d \delta^{*}}
$$


The term $d M_{o} / d \delta^{*}$ is easy to calculate on the domain $M_{o}=M_{c}$. Noting that that $M_{o}=M_{c}=0$ when $\delta^{*}=\delta_{\max }$, due to the equilibrium condition, and $L_{a}=L$, we simply get

$$
\frac{d F}{d \delta^{*}}\left(\delta^{*}=\delta_{\max }\right)=\frac{1}{L} \frac{d M_{c}}{d \delta^{*}}
$$

which is easily calculated, since $\left(d M_{c} / d \delta^{*}\right)(\delta)=-M g\left(X_{G} \sin \delta+Y_{G} \cos \delta\right)$.

The force coefficient $C_{F_{\max }}$ is introduced to adapt the force prediction to the measurements (Litrico et al., 2005):

$$
\frac{d F}{d \delta^{*}}\left(\delta^{*}=\delta_{\max }\right)=C_{F_{\max }} \frac{1}{L} \frac{d M_{c}}{d \delta^{*}}
$$

The value of $C_{F_{\max }}$ is linked to the pressure field and its influence on the lever arm $L_{a}$, which is calculated as in hydrostatic conditions.

Litrico et al. (2005) proposed empirical relationships linking the values of $C_{F_{0}}$ and $C_{F_{\max }}$ to $\Phi$ 's angles. In practice, these coefficients can be tuned when measurements of $h_{0}$ and $Q$ are available in free flow. Table 2 gives the values of the tuned coefficients $C_{F_{0}}$ and $C_{F_{\max }}$ obtained for both gates in free flow.

\section{Interpolation}

To determine the force exerted on the upstream side of plate, a cubic interpolation is done between $\delta^{*}=0$ and $\delta^{*}=\delta_{\max }$, using the calculated values of the force and its derivatives in 0 and $\delta_{\max }$ (see appendix and discharge law calculation for details):

$$
F\left(\delta^{*}\right)=F(0)+c_{1} \delta^{*}+c_{2} \delta^{* 2}+c_{3} \delta^{* 3}
$$

This is the same expression as in free flow. However, the submergence modifies the force at equilibrium as discussed below.

\section{Effect of Submergence}

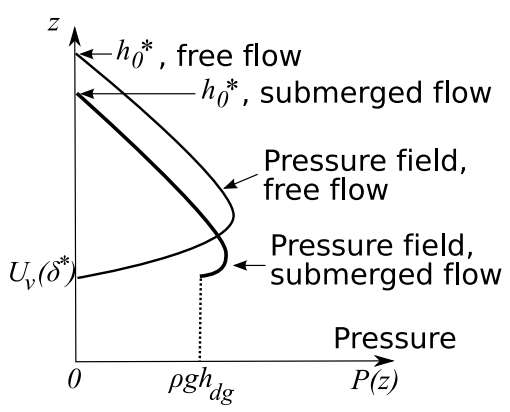

Figure 9: Pressure field on the gate for a given equilibrium angle $\delta^{*}$

The downstream water level $h_{2}$ does not appear in any of the previous equations when calculating the force, which means that it is assumed that the force at equilibrium is unchanged in submerged conditions. This assumption is not exactly true, since the submerged condition modifies the pressure field on the upstream face of the gate: at the bottom of the gate, the relative pressure is null in case of free flow, but equals $\rho g h_{d g}$ otherwise, where $h_{d g}=h_{2}-U_{v}$ denotes the downstream water height above the gate bottom lip (see Fig. 9). Therefore, for a given water level $h_{0}$ and a given equilibrium opening angle $\delta^{*}$, the lever arm is greater in 
submerged flow than in free flow, as well as the pressure force exerted on the gate. This means that, for a given equilibrium angle $\delta^{*}$, the equilibrium water level $h_{0}^{*}$ must be lower in submerged flow than in free flow. When the equilibrium angle is known, the equilibrium force is calculated as follows:

$$
F\left(\delta^{*}\right)=\frac{M_{c}\left(\delta^{*}\right)}{L_{a}\left(\delta^{*}, h_{0}^{*}\right)}
$$

with

$$
L_{a}\left(\delta^{*}, h_{0}^{*}\right)=L-\frac{h_{0}^{*}-U_{v}\left(\delta^{*}\right)}{3 \cos \left(\delta^{*}\right)}
$$

Since $L_{a}$ is greater in submerged flow than in free flow, for a certain value of $\delta^{*}$, the corresponding force at equilibrium $F\left(\delta^{*}\right)$ is smaller. The ratio of the equilibrium force in submerged flow to the equilibrium force in free flow, denoted $\varphi$, should therefore depend on the shape of the pressure field, $\varphi$ being close to 1 at low submergence (when $h_{2} \ll h_{0}$ ) and minimum at high submergence (when $h_{2} / h_{0} \rightarrow 1$ ).

The variation of $\varphi$ (measured force in submerged flow to the model of the force in free flow) with respect to the submergence ratio $s=h_{2} / h_{0}$ is plotted for both gates on Fig. 10. It can be observed that $\varphi$ decreases down to 0.93 (ENSAM gate) and 0.85 (Delft gate) when $h_{2} / h_{0} \rightarrow 1$. The relationship is slightly different for both gates, but no clear influence of the discharge nor the angle $\Phi$ could be observed.

Therefore, a simple power law is adjusted by a least square method, imposing $\varphi=1$ when $h_{2} \leq 0$ :

$$
\begin{aligned}
& s>0: \varphi(s)=1-\beta s^{b} \\
& s \leq 0: \varphi(s)=1
\end{aligned}
$$

The best fitting is obtained with $\beta=0.08$ and $b=1.3$. To take account of the different behavior of both gates, the coefficient $\beta$ is adjusted for each gate. This parameter represents the maximum variation of $F\left(\delta^{*}\right)$ between free flow and submerged flow. We obtain $\beta=0.12$ for Delft gate and $\beta=0.08$ for ENSAM gate. This means that the submergence effect can modify the force (for a given $\delta^{*}$ ) up to $8 \%$ for ENSAM gate and $12 \%$ for Delft gate. The values of $\beta$ will be analyzed in the discussion section.

Table 2: Force coefficients $C_{F_{0}}, C_{F_{\max }}$ and $\beta$

\begin{tabular}{lccc}
\hline \hline Gate, position & $C_{F_{0}}$ & $C_{F_{\max }}$ & $\beta$ \\
\hline ENSAM, $\Phi=38^{\circ}$ & 0.9 & 1.0 & 0.08 \\
ENSAM, $\Phi=45^{\circ}$ & 0.8 & 1.15 & 0.08 \\
ENSAM, $\Phi=58^{\circ}$ & 0.7 & 1.2 & 0.08 \\
Delft, $\Phi=35^{\circ}$ & 1.5 & 1.4 & 0.12 \\
Delft, $\Phi=45^{\circ}$ & 1.0 & 1.2 & 0.12 \\
Delft, $\Phi=55^{\circ}$ & 0.7 & 1.2 & 0.12 \\
\hline
\end{tabular}

\section{Opening Moment}

The opening moment is finally obtained as:

$$
M_{o}\left(h_{0}, h_{2}, \delta^{*}\right)=L_{a}\left(h_{0}, \delta^{*}\right) \varphi\left(h_{2} / h_{0}\right) F\left(\delta^{*}\right)
$$

with $L_{a}\left(h_{0}, \delta^{*}\right)$ given by Eq. (35), the force in free flow $F\left(\delta^{*}\right)$ given by the cubic interpolation (Eq. (42)) and $\varphi\left(h_{0} / h_{2}\right)$ given by Eq. (45) and (46). 


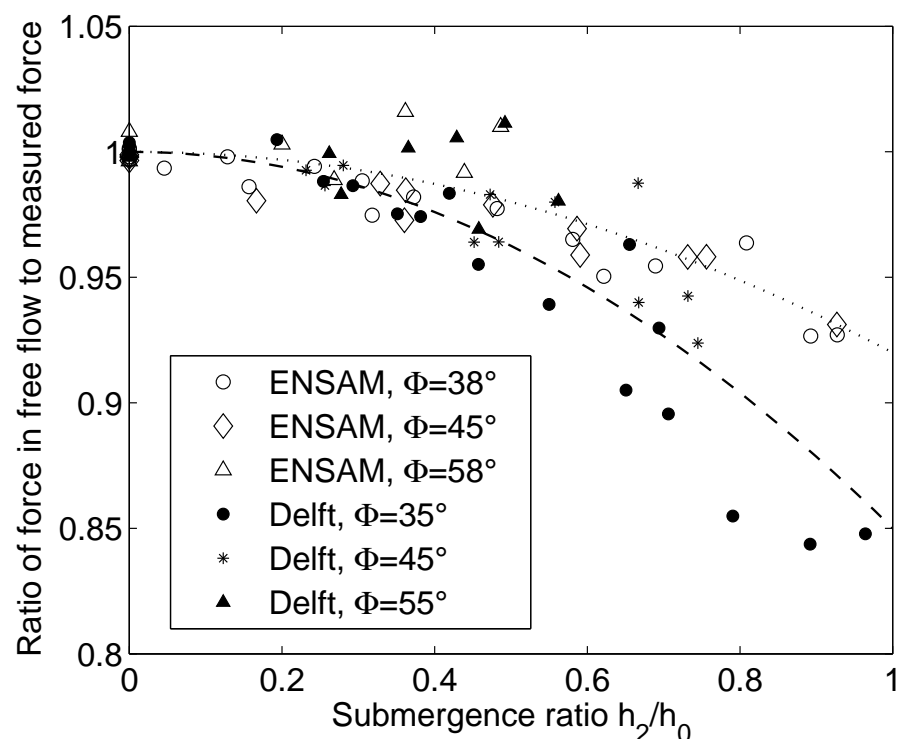

Figure 10: Ratio of the measured force to the force in free flow, ENSAM and Delft gates. The dashed line is fitted on Delft gate, the dotted line on ENSAM gate.

\section{Validation of the Equilibrium Model}

For each measured vector $\left(Q, h_{0}, h_{2}, \delta^{*}\right)$, the measured opening moment is assessed thanks to the closing moment calculation (Eq. (34)), since $M_{0}=M_{c}$ at equilibrium.

The simulated opening moment is calculated using Eq. 47. For each gate configuration, we only have three parameters for the model of the force: $C_{F_{0}}, C_{F_{\max }}$ and $\beta$, the value of $\beta$ being independent on $\Phi$. The calibrated values of these coefficients are given in table 2 . The correspondence between simulated and measured moments is excellent as shown on Fig. 11.

\section{Full Model of the Vlugter Gate}

Now both discharge and equilibrium formulae are combined to determine the opening angle at equilibrium and the corresponding upstream water level $h_{0}$.

For given discharge and downstream water level, the computation is made by a bisection method on $h_{0}$ :

1. Initialization: Determine $h_{0_{\min }}=h_{w}$, which is the water level in weir condition computed by inverting the weir discharge equation (Eq. 16 and 19); this is the minimum water level for given values of $Q$ and $h_{0}$. A maximum value of $h_{0}$ is for example $h_{0_{\max }}=L+p$ $(\delta=\pi / 2)$. Set $\varepsilon=10^{-4}$ N.m (maximum difference between opening and closing moment at equilibrium).

2. Compute the opening $\delta$ necessary to have the discharge $Q$ with the upstream level $h_{0}$, downstream level $h_{2}$, using the discharge equation (32).

3. Compute the opening moment $M_{o}\left(h_{0}, h_{2}, \delta\right)$ using Eq. (47) and the closing moment $M_{c}(\delta)$ using Eq. (34). 


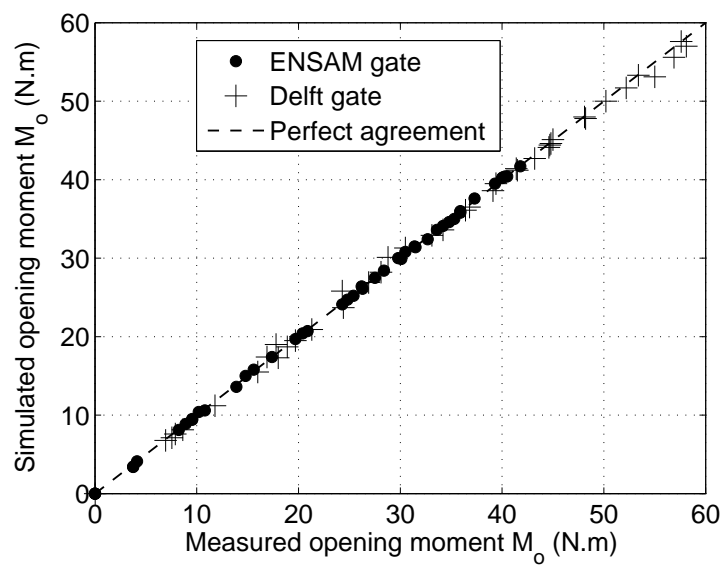

Figure 11: Simulated versus measured moment, ENSAM and Delft gates. RMSE (Root mean square error $)=0.5 \mathrm{Nm}$.

4. Compute the difference between opening and closing moment $e=M_{o}\left(h_{0}, h_{2}, \delta\right)-M_{c}(\delta)$. If $|e|<\varepsilon, \delta$ is the angle of equilibrium and $h_{0}$ is the upstream level for equilibrium (end of the computation), else

$$
\begin{aligned}
& \text { - if } e>0 \text { then do } h_{0_{\min }}=h_{0} \\
& \text { - if } e<0 \text { then do } h_{0_{\max }}=h_{0}
\end{aligned}
$$

5. Do $h_{0}=\left(h_{0_{\min }}+h_{0_{\max }}\right) / 2$ and go to step 2 .

\section{Prediction of Upstream Water Levels}

The equilibrium state is now analyzed by comparing the simulated water levels with the measured ones. The predicted values result from the combination of the discharge law and the equilibrium law. Figures 12 and 13 show the variation of $h_{0}$ with $h_{2}$ for $Q=30 \mathrm{l} / \mathrm{s}$ (ENSAM gate) and $Q=90 \mathrm{l} / \mathrm{s}$ (Delft gate) with the different counterweight positions. We observe that the model can simulate correctly the decrease of $h_{0}$ with $h_{2}$.

The complete results are presented on figure 14 for ENSAM and Delft gates. The prediction is rather good; $84 \%$ of the simulated water levels deviates from less than $5 \%$ of the measured values and all but one deviate by less than $10 \%$. The root mean square error (RMSE) is around 1 centimeter for all simulations.

The maximum error is observed for high submergence ratios. The performance of the model could be improved by tuning the model parameters acting in submerged conditions. For instance, different values of $\beta$ could be chosen for each counterweight position, which slightly improves the gate prediction.

Finally, the model is applied to the original data provided by Vlugter (1940). The opening angles are not reported and cannot be used for the calculation of the opening moment and force at equilibrium. Therefore, the force coefficients given by the heuristic approach of Litrico et al. (2005) are used, and the submergence coefficient is tuned to fit the model on the observed measurements. An optimal value of $\beta=0.06$ is obtained, which is very close to those obtained for ENSAM and Delft gates. We can conclude that this coefficient is only slightly affected by scale effects. In this case, the calibration gives a maximum error of $3 \mathrm{~cm}$ (less than $2 \%$ of 


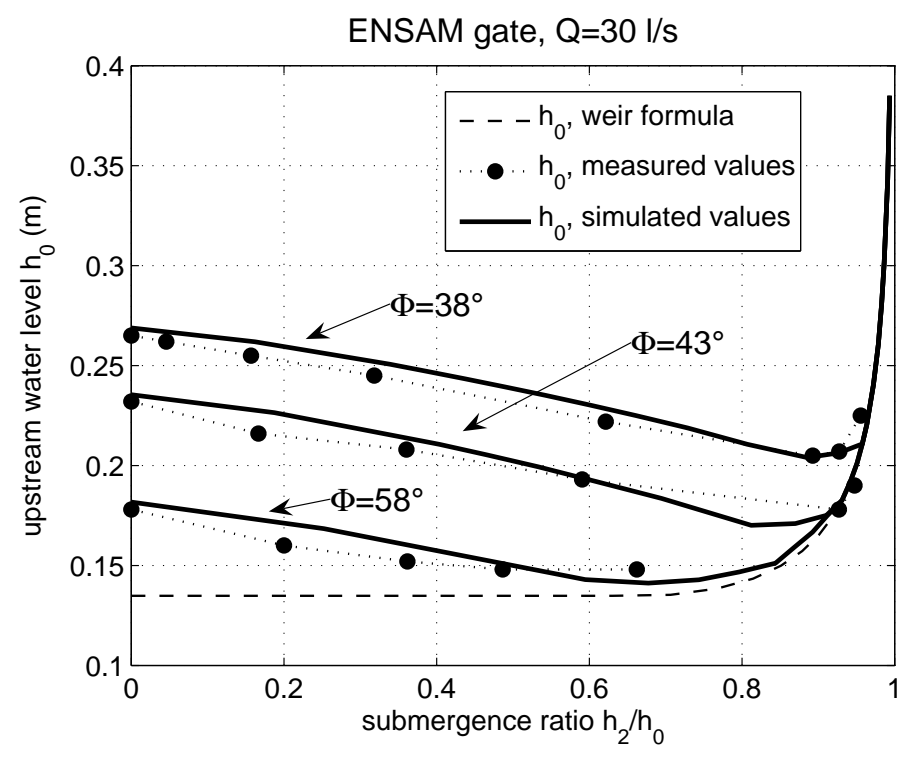

Figure 12: ENSAM gate, simulated and measured upstream levels with respect to the submergence ratio $h_{2} / h_{0}-$ discharge $=30 \mathrm{l} / \mathrm{s}$. RMSE $=0.0106 \mathrm{~m}$.

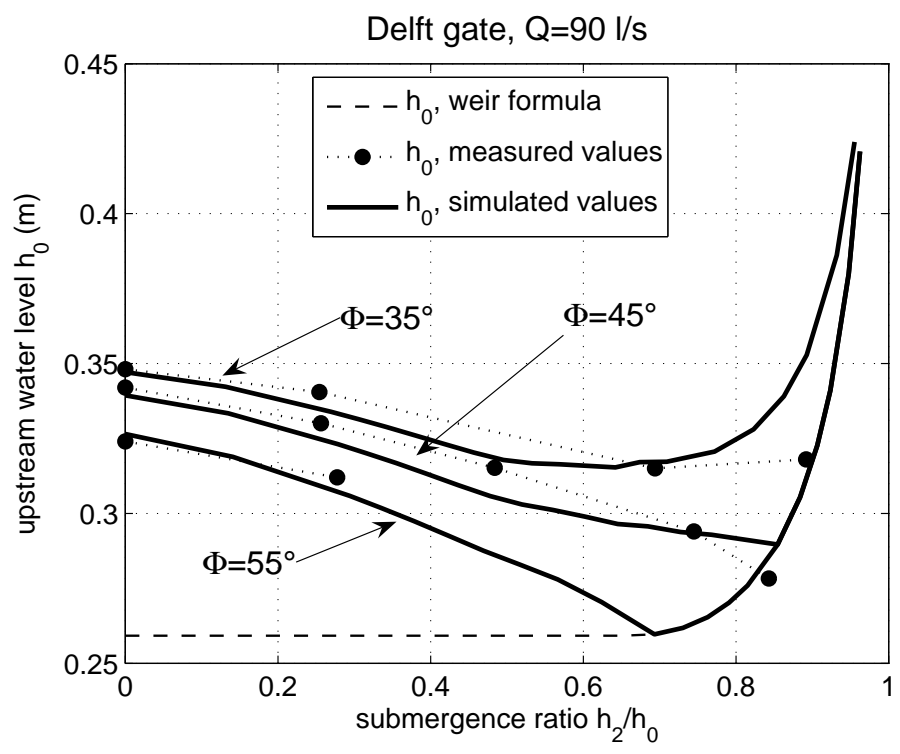

Figure 13: Delft gate, simulated and measured upstream levels with respect to the submergence ratio $h_{2} / h_{0}-$ discharge $=90 \mathrm{l} / \mathrm{s}$. RMSE $=0.009 \mathrm{~m}$. 


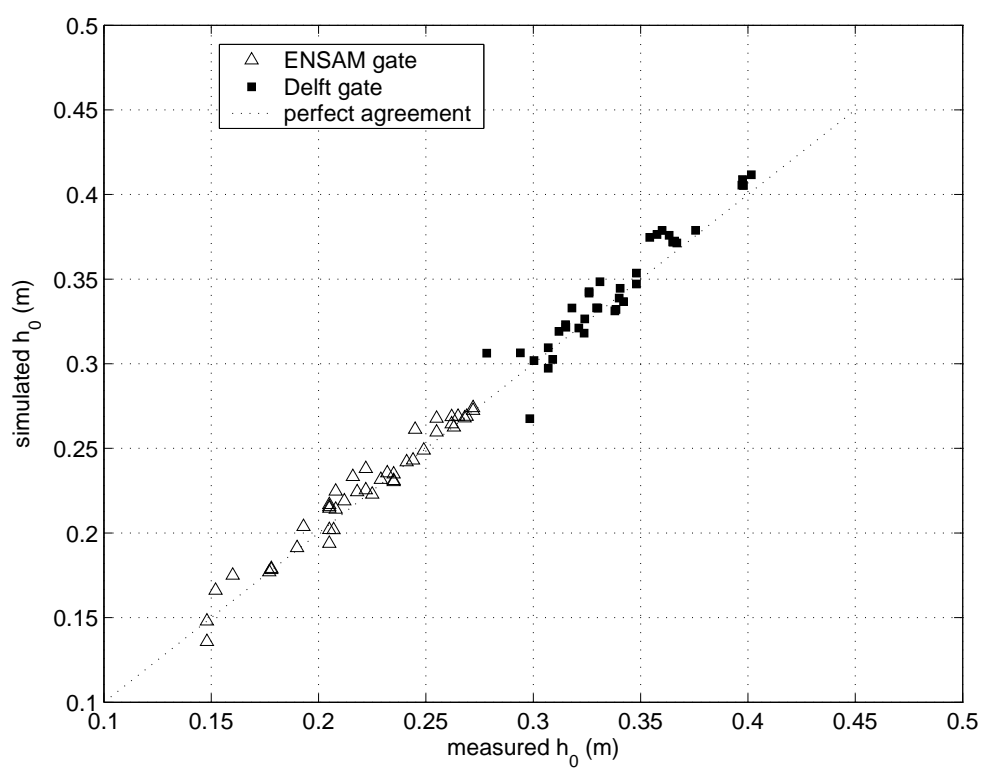

Figure 14: Simulated versus measured upstream water level, ENSAM and Delft gates

the measured upstream level) between predicted and measured values (figure 15). Using the coefficients obtained for Delft and ENSAM gates yields a larger error $(7 \mathrm{~cm}$, which corresponds to $5 \%$ ) but still acceptable.

\section{Prediction of Opening Angles at Equilibrium}

The opening angles are correctly simulated in free flow. When the submergence ratio $s$ increases, the gate opening increases due to the head-opening-discharge relationship. Fig. 8 showed larger errors on the gate opening $\delta$ for large values of $\delta / \delta_{\max }$, due to the small sensitivity of the discharge with respect to $\delta$ in this domain. The same discrepancy is observed when simulating the opening angle at equilibrium. This discrepancy is mainly due to the discharge relationship, since the model of the force is very accurate.

Fig. 16 depicts the variation of $\delta^{*}$ with the submergence ratio $s=h_{2} / h_{0}$. The simulated values result from the complete calculation of $h_{0}$ and $\delta^{*}$. Again, it can be observed that the prediction is accurate unless for large angles $\left(\delta^{*}\right.$ close to $\left.\delta_{\max }\right)$, which corresponds to large submergence ratios.

\section{Discussion}

The approach presented here is rather accurate to model the gate behavior in submerged conditions. Compared to free flow, the discharge formula needs to include a reduction factor $S$, expressed as a function of the submergence ratio $h_{2} / h_{0}$. This reduction factor uses calibrated coefficients, but standard parameters gave satisfactory results on the studied gates.

The effect of submergence on the force at equilibrium is simulated using a parameter $\beta$ which accounts for the pressure field modification between free flow and submerged flow. The pressure field implies modifications of the pressure force and on the lever arm position. Both effects can be analyzed through the following non-dimensional numbers, characteristic of the gate geometries: 


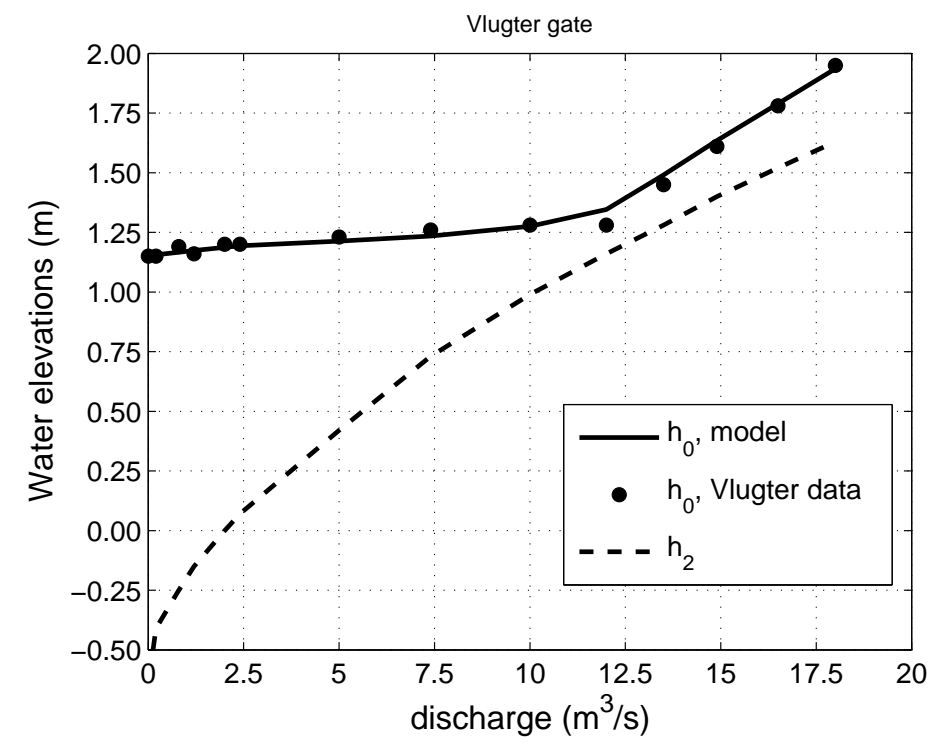

Figure 15: Vlugter gate, original data - comparison of model and data

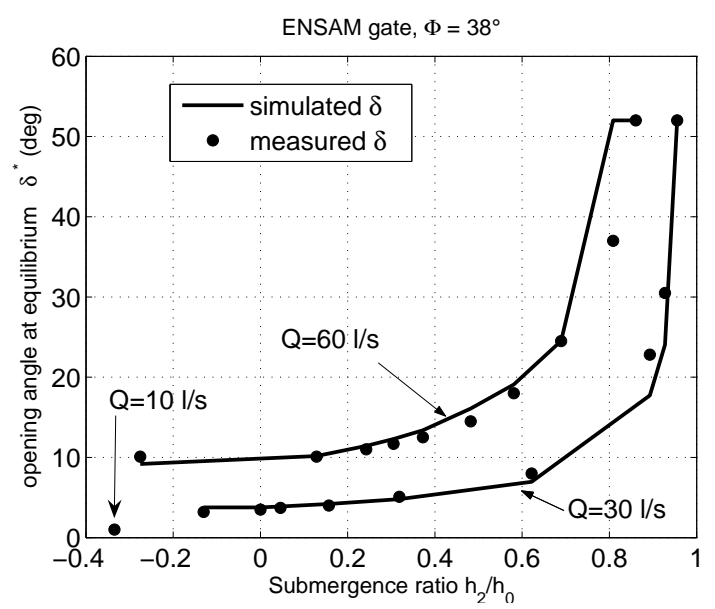

Figure 16: Simulated angle, ENSAM gate, $\Phi=38^{\circ}$. Due to gate oscillations, only one measurement is available for $Q=10 \mathrm{l} / \mathrm{s}$; in this case, the predicted angle merges into the measured one. 
- $r_{1}=h_{d} / B_{g}$, ratio of design upstream level to gate width: when $r_{1}$ is large, the side effects are significant. In this case, the difference between the actual pressure field and the hydrostatic field should be high. Larger values of $\beta$ should be observed.

- $r_{2}=\frac{h_{d}}{L}$, ratio of design water level to gate height: the lever arm $L_{a}$ is determined by the pressure field, although it is calculated like in hydrostatic conditions. Actually, the incertitude is all the higher as the water column on the upstream side of the gate is high, say $r_{2}$ is close to 1 . On the contrary, small values of $r_{2}$ imply that $L_{a} \simeq L$ whether the pressure field is hydrostatic or not.

These ratios are calculated for each studied gate (Table 3). Although the number of studied systems is not sufficient to establish quantitative relationships, we can observe a certain coherence in the correction factors $\beta$. The higher values of $\beta$ correspond to Delft gate, where the side effects are significant ( $r_{1}$ high) and the design level is high $\left(r_{2}\right.$ high). The Vlugter gate is rather wide and the side effect are negligible. In this case, we can observe a rather small value of the correction factor $\beta$. ENSAM gate has slightly lower values of $r_{1}$ and $r_{2}$ than Delft gate which is consistent with the variation of $\beta$.

Table 3: Gate characteristics and $\beta$ parameter

\begin{tabular}{lccccccc}
\hline \hline Gate & $\Phi(\mathrm{deg})$ & $h_{d}(\mathrm{~m})$ & $B_{g}(\mathrm{~m})$ & $L(\mathrm{~m})$ & $r_{1}=h_{d} / B_{g}$ & $r_{2}=h_{d} / L$ & $\beta$ \\
\hline Delft & {$[35 ; 45 ; 55]$} & 0.30 & 0.40 & 0.47 & 0.75 & 0.64 & 0.12 \\
ENSAM & {$[38 ; 45 ; 58]$} & {$[0.27 ; 0.23 ; 0.18]$} & 0.38 & 0.42 & {$[0.71 ; 0.61 ; 0.47]$} & {$[0.64 ; 0.55 ; 0.43]$} & 0.08 \\
Vlugter & 45 & 1.15 & 6.00 & 1.50 & 0.19 & 0.77 & 0.06 \\
\hline \hline
\end{tabular}

Finally, the sensitivity to this parameter is analyzed (Fig. 17). The upstream water level is simulated for different values of $\beta$, from $\beta=0$ (no effect of submergence taken into account) to $\beta=0.12$ (value obtained for Delft gate). It can be observed that the upstream water level can decrease when $h_{2}$ increases only when the effect of submergence on the force is taken into account $(\beta \neq 0)$. Exaggerated values of $\beta$ exaggerate the decrease of $h_{0}$, and lead to an error of 3 centimeters at high submergence ratios (15\%).

\section{Conclusion}

This paper derives a model of the Vlugter gate behaving under submerged conditions. The difficulty to model such systems is to simulate the transition regime between the undershot gate behavior (small gate opening) and the weir behavior (large opening).

The proposed model combines a discharge law, based on the interpolation between a undershot gate behavior and a weir behavior, and an equilibrium law based on the trajectory at equilibrium of the force exerted on the gate.

The discharge formula was validated on two small-scale gates and comprises only two classical parameters (discharge coefficients for an undershot gate and a broad-crested weir). This formula can be applied to any rectangular flap gate.

In free flow, the equilibrium relationship uses two parameters depending on the position of the center of gravity. To take account of the submergence effect, one tuning parameter is introduced.

Coupling the discharge law with the equilibrium law, the model is able to accurately simulate the upstream water level $h_{0}$ and the gate opening at equilibrium $\delta^{*}$ for a given discharge $Q$ and a downstream water level $h_{2}$. 


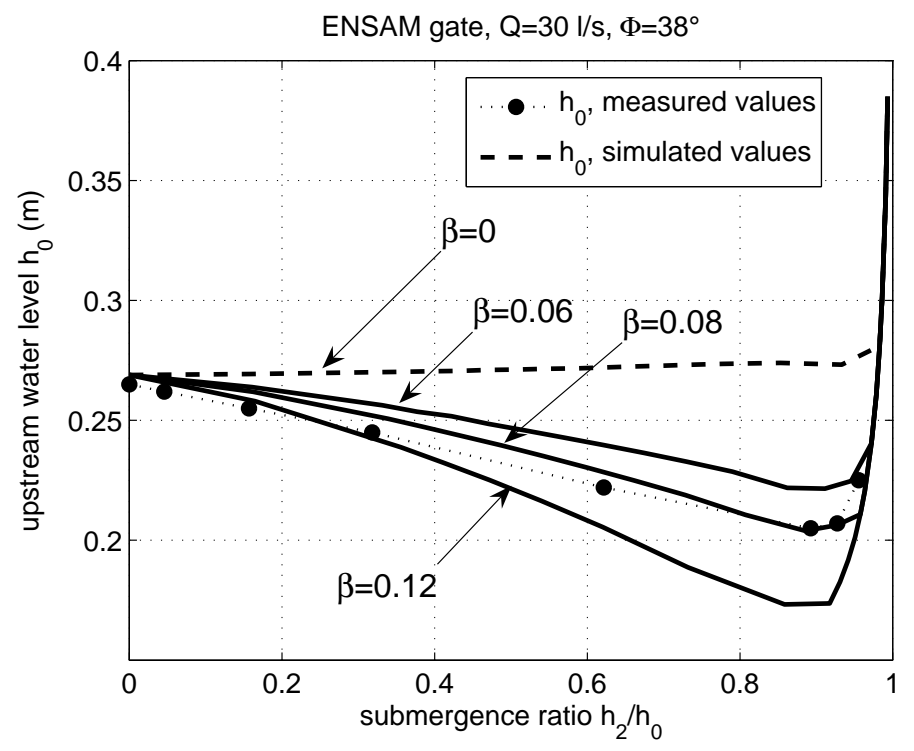

Figure 17: ENSAM gate, $\Phi=38^{\circ}$; influence of parameter $\beta$ on simulated water levels

The model has been validated on a set of data collected on two small-scale gates, and on the original Vlugter gate data (Vlugter, 1940), with a very satisfying result. This model enables to accurately predict the stationary behavior of the gate.

The proposed model could be used to evaluate the impact of design parameters on the gate performance in terms of water level control.

\section{Acknowledgements}

The experimental setup was built by Paul Oullier and Gérard Alvarez, technical assistants of the hydraulic laboratory of ENSAM, whose help is gratefully acknowledged. We would also like to thank Prof. R. Brouwer from Delft University of Technology who kindly provided the data from de Graaff's master's thesis.

\section{Appendix}

\section{Interpolation of a Cubic Curve}

Let $f(\delta)$ denote the function defined for $\delta \in\left[0, \delta_{w}\right]$ that verifies the following interpolation conditions:

- $f(0)=f_{0}$,

- $f\left(\delta_{w}\right)=f_{w}$,

- $\frac{d f}{d \delta}(0)=d f_{0}$,

- $\frac{d f}{d \delta}\left(\delta_{w}\right)=d f_{w}$.

where $f_{0}, f_{w}, d f_{0}, d f_{w}$ are real numbers giving the values of the function and its derivative at 0 and $\delta_{w}$. 
$f(\delta)$ is parameterized as a cubic polynomial:

$$
f(\delta)=c_{0}+c_{1} \delta+c_{2} \delta^{2}+c_{3} \delta^{3}
$$

The four interpolation conditions provide a linear system of four equations. Solving for $c_{0}, c_{1}$, $c_{2}$ and $c_{3}$ gives:

$$
\begin{aligned}
& c_{0}=f_{0} \\
& c_{1}=d f_{0} \\
& c_{2}=3 \frac{f_{w}-f_{0}}{\delta_{w}^{2}}-\frac{2 d f_{0}+d f_{w}}{\delta_{w}} \\
& c_{3}=2 \frac{f_{0}-f_{w}}{\delta_{w}^{3}}+\frac{d f_{0}+d f_{w}}{\delta_{w}^{2}}
\end{aligned}
$$

\section{Notations}

The following notations are used in this paper:

$\beta=$ correction factor on the force at equilibrium

$\delta=$ angle of opening (rad)

$\delta_{w}=$ angle of opening corresponding to the flow over the weir (rad)

$\delta_{\max }=$ maximum angle of opening corresponding to the unstable equilibrium ( $\mathrm{rad}$ )

$\Phi=$ angle $(O x, O G)(\mathrm{rad})$

$\rho=$ density of water $\left(1000 \mathrm{~kg} / \mathrm{m}^{3}\right)$

$B_{c}=$ canal width, downstream pool (m)

$B_{g}=$ gate width $(\mathrm{m})$

$B_{w}=$ weir width $(\mathrm{m})$

$C_{F_{0}}$ : tuning coefficient

$C_{F_{\max }}$ : tuning coefficient

$C_{c}$ : contraction coefficient

$C_{u}$ : discharge coefficient for the flow under the gate

$C_{w}, C_{w_{0}}:$ Weir discharge coefficient

$F=$ opening force $(\mathrm{N})$

$g=$ gravitational acceleration $\left(9.81 \mathrm{~m} / \mathrm{s}^{2}\right)$

$H_{0}=$ upstream head $(\mathrm{m})$

$H_{2}=$ downstream head $(\mathrm{m})$

$h_{0}=$ upstream water level $(\mathrm{m})$

$h_{1}=$ hydrostatic head in the contracted section $(\mathrm{m})$

$h_{2}=$ downstream water level $(\mathrm{m})$

$h_{d}=$ design upstream water level $(\mathrm{m})$

$h_{w u}=$ upstream height of the weir $(\mathrm{m})$

$h_{w d}=$ downstream height of the weir $(\mathrm{m})$

$h_{w}=$ upstream water level in weir behavior $(\mathrm{m})$

$L=$ gate height $(\mathrm{m})$

$L_{a}=$ lever arm for the opening moment calculation (m)

$L_{w}$ : weir length $(\mathrm{m})$

$M_{G}=$ mass of the gate without counterweight $(\mathrm{kg})$

$M_{W}=$ mass of the counterweight $(\mathrm{kg})$

$M=M_{W}+M_{G}=$ total mass of the gate and counterweight $(\mathrm{kg})$ 
$M_{o}=$ opening moment $(\mathrm{Nm})$

$M_{c}=$ closing moment $(\mathrm{Nm})$

$p=$ lever arm from hinge point to gate $(\mathrm{m})$

$Q=$ total discharge $\left(\mathrm{m}^{3} / \mathrm{s}\right)$

$Q_{r}=$ residual discharge at $\delta=0\left(\mathrm{~m}^{3} / \mathrm{s}\right)$

$Q_{w}=$ discharge in weir position $\left(\mathrm{m}^{3} / \mathrm{s}\right)$

$Q_{w f}=$ discharge in weir position, free flow $\left(\mathrm{m}^{3} / \mathrm{s}\right)$

$Q_{\text {side }}=$ side discharge $\left(\mathrm{m}^{3} / \mathrm{s}\right)$

$Q_{\text {under }}=$ discharge under the gate $\left(\mathrm{m}^{3} / \mathrm{s}\right)$

$r_{1}=h_{d} / B_{g}=$ ratio of design upstream level to gate width

$r_{2}=\frac{h_{d}}{L}$, ratio of design water level to gate height

$s=h_{2} / h_{0}=$ submergence ratio

$S_{w}=Q_{w} / Q_{w f}=$ correction factor on the weir discharge, due to submergence

$U=$ gate opening $(\mathrm{m})$

$U_{h}=$ horizontal gate opening $(\mathrm{m})$

$U_{v}=$ vertical gate opening $(\mathrm{m})$

$V_{u}=$ mean velocity at the brink $(\mathrm{m} / \mathrm{s})$

$x=$ horizontal distance from the weir downstream brink $(\mathrm{m})$

$X_{G}=$ horizontal distance of the center of gravity from the pivot point $(\mathrm{m})$

$y_{L}=$ modular limit of a weir (maximum value of $s$ for free flow)

$Z_{G}=$ vertical distance of the center of gravity from the pivot point $(\mathrm{m})$

$z=$ vertical distance from the weir crest $(\mathrm{m})$

ENSAM $=$ Ecole Nationale Supérieure Agronomique de Montpellier

HVIP = Hadejia Valley Irrigation Project

\section{References}

Bos (1989). Discharge measurement structures. 3rd edition, ILRI, Wageningen, The Netherlands

Brants, M. (1996). Automatic gates facilitate water management in Punggur Utara irrigation project. In ICID, editor, 16th International Congress on Irrigation and Drainage, Cairo, Egypt.

Burt, C., Angold, R., Lehmkuhl, M., and Styles, S. (2001). Flap gate design for automatic upstream canal water level control. J. of Irrigation and Drainage Engineering, 127(2):84-91.

Brouwer, R. (1987). Design and application of automatic check gate for tertiary turnouts. In 13th ICID Congress, pages 671-683, Rabat, Morroco.

Cunge, J.A., Holly, F.M., Vervey, A. (1980). Practical aspects of computational river hydraulics. Pitman. 420p.

Davis, A., Jacob, P., and Ellett, G. (1999). Estimating trajectory of free overfall nappe. J. of Hydraulic Engineering, 125(1):79-82.

de Graaff, B. (1998). Stability analysis of the Vlugter gate. Master's thesis, Delft University of Technology, Faculty of Civil Engineering and Geosciences. 
Hager, W. (1983). Hydraulics of plane free overfall. J. of Hydraulic Engineering, 109(12):16831697.

Hager, W. and Schwalt, M. (1994). Broad-crested weir. J. Irrig. Drain. Engrg, 109(12):16831697.

Litrico, X., Belaud, G., Baume, J.-P., and Ribot-Bruno, J. (2005). Hydraulic modeling of an automatic upstream water-level control gate. J. Irrig. Drain. Engrg., 131(2):176-189.

Sinniger, R. and Hager, W. (1989). Constructions hydrauliques. Traité de Génie Civil, Vol. 15. Presses Polytechniques Romandes, 439p. (in French).

Vlugter, H. (1940). Over zelfwerkende peilregelaars bij den waterstaat in nederlandsch-indie. De ingenieur in Nederlandsch-Indie, (6):84-93. (in Dutch).

USDA (1973). Submerged weir flow. Soil Conservation Service, design note No 15 